\title{
AVALIAÇÃO DO POTENCIAL DA SEMENTE DE MORINGA OLEIFERA PARA OBTENÇÃO DE COAGULANTE E ÓLEO UTILIZANDO ULTRA-SOM
}

\author{
M. O. SILVA ${ }^{1}$, A. T. A. BAPTISTA ${ }^{2}$, L. A. M. PINTO ${ }^{2}$, E.C.D. SENNA ${ }^{1}$, R. BERGAMASCO ${ }^{1}$, \\ A. M. S. VIEIRA ${ }^{2}$ \\ ${ }^{1}$ Universidade Estadual de Maringá, Departamento de Engenharia Química \\ ${ }^{2}$ Universidade Estadual de Maringá, Departamento de Engenharia de Alimentos \\ e-mail: aline_takaoka17@ @otmail.com
}

\begin{abstract}
RESUMO
Os produtos resultantes da árvore Moringa oleifera (Moringa) apresentam um vasto potencial tecnológico, pois abrangem positivamente vários aspectos, tais como baixo custo, alto valor nutricional, óleo rico em ácido oleico e tocoferóis, alto poder coagulante e fácil adaptação em climas tropicais. Estudos relatam que mesmo após a retirada do óleo da semente, a torta resultante continua com suas propriedades coagulantes, ou seja, é possivel obter o óleo e utilizar a torta para obtenção do coagulante natural utilizado no tratamento de águas. O objetivo do presente estudo foi avaliar o potencial da semente de Moringa para obtenção de óleo de boa qualidade utilizando um processo químico empregando o ultrassom e o hexano como solvente, e o processo mecânico. Foram avaliados a utilização do farelo desengordurado nos dois processos de extração como agente coagulante em comparação ao farelo integral. Os resultados mostram um alto teor de óleo (33\%) em processos químicos de extração em comparação a processos mecânicos $(26 \%)$, sendo que o óleo apresentou boa qualidade, com índice de acidez de 0,54 e 0,44 (\% em ácido oleico), respectivamente para extração com ultrassom e prensa. Quanto ao potencial coagulante a torta desengordurada utilizando o ultrassom apresentou alto potencial de remoção de cor $(92,45 \%)$ e turbidez $(93,55 \%)$ da água superficial além de adicionar menor quantidade de matéria orgânica na água tratada quando comparada com coagulante produzido pela semente integral.
\end{abstract}

\section{INTRODUÇÃO}

As sementes de Moringa oleifera Lam. (Moringa) apresentam-se ricas em tocoferóis e com baixo conteúdo em ácidos graxos poliinsaturados $(<1 \%)$, o que confere ao óleo notável estabilidade oxidativa. A estabilidade oxidativa do óleo da semente de Moringa é maior que outros óleos ricos em ácido oleico, sendo estudada a possibilidade da adição do óleo de Moringa em outros óleos, como o de girassol, com o intuito de aumentar a sua estabilidade. A composição em ácidos graxos do óleo da semente da Moringa é semelhante ao azeite de oliva, principalmente no teor de ácidos graxos monoinsaturados se destacando o teor de ácido oleico. Estudos relatam também a utilização do óleo na fabricação de cosméticos onde atua como agente emoliente e também sua aplicação como biodiesel (ABDULKARIM et al., 2005; ANWAR et al., 2007; AYERZA, 2011).

Outro aspecto de interesse na semente de Moringa se diz respeito a sua eficiência 
comprovada por vários autores (MADRONA et al 2010; VIEIRA et al., 2010; NISHI et al., 2011). Segundo Ndabigengesere e Narasiah (1998), as sementes de Moringa são uma alternativa viável de agente coagulante em substituição aos sais de alumínio, que são utilizados no tratamento de água em todo o mundo. Em recente estudo avaliando o potencial coagulante da semente integral e a torta da extração, também denominado farelo desengordurado, obteve-se nas mesmas concentrações de coagulantes valores mais baixos de turbidez, tempo ótimo de estabilização (15-60 minutos versus $60-120$ para o farelo integral) e diminuição da matéria orgânica em relação à semente integral de Moringa (KABORE et al., 2013). Desta forma o presente trabalho teve como objetivo avaliar o potencial da semente de Moringa para obtenção de óleo e a utilização do farelo desengordurado como agente coagulante em água superficial.

\section{MATERIAIS E MÉTODOS}

As sementes de Moringa foram obtidas da Universidade Estadual de Sergipe, foram separadas das cascas e selecionadas de modo a serem utilizadas apenas sementes sadias. A água utilizada nos ensaios de coagulação/floculação foi proveniente da bacia do Rio Pirapó, coletada na Companhia de Saneamento do Paraná - SANEPAR, localizada na cidade de Maringá - Paraná.

\subsection{Extração do Óleo}

O óleo de Moringa foi extraído por dois métodos, um químico utilizando solvente e o aparelho ultrassom, e outro físico, utilizando prensagem. Para as extrações com ultrassom, foi utilizado uma quantidade de amostra de 5 g, um banho ultrassônico UltraCleaner 800 com uma frequência de $40 \mathrm{kHz}$ e erlenmeyers de $250 \mathrm{~mL}$, com boca esmerilhada. Mantevese a água do banho do equipamento a uma temperatura de $25^{\circ} \mathrm{C}$, conforme Menezes et al. (2013).

A melhor condição de extração do óleo foi obtida por meio de um delineamento fatorial $3^{2}$ (3 tempos de extração: 30, 60 e 90 minutos; 3 proporções de massa de solvente: volume de solvente de 1:8 1:12 e 1:16 ). Neste caso o solvente utilizado foi o hexano. Para isso, foram realizados a análise de variância (ANOVA) e o teste de comparação de médias, teste Tukey, com 95\% de confiança, sendo significativo um p-valor < 0,05 , para verificar as diferenças significativas, através do programa estatístico STATISTICA, versão 8.0.

Para a extração mecânica do óleo foi utilizada uma prensa hidráulica 30T Bovenau, com pressão máxima de $66,57 \mathrm{MPa}$, sendo utilizada a pressão de $26,63 \mathrm{MPa}$ e aproximadamente $75 \mathrm{~g}$ de semente de Moringa. A prensagem da amostra ocorreu por um período de 2 horas, tempo em que não foi observado variação no teor de óleo extraído (SILVA et al., 2013).

As amostras de óleo, após processos de extração, foram armazenadas em recipientes de vidro âmbar a temperatura de aproximadamente $-18^{\circ} \mathrm{C}$ para evitar posteriores alterações oxidativas, e descongeladas apenas no momento das análises. Para a avaliação da qualidade do óleo foram realizadas as análises de índice de acidez e índice de saponificação, segundo a AOAC (1998), nas amostras do óleo de Moringa extraído por prensa e por ultrassom, na melhor condição de extração.

Todas as análises das amostras foram realizadas em triplicata. Os resultados foram analisados no software Statistica $8.0^{\circledR}$ aplicando a ANOVA e utilizando o teste $\mathrm{T}$ de Tukey com 5\% de significância.

\subsection{Obtenção do Coagulante Salino}

Para a obtenção dos coagulantes foram utilizados a semente integral e os farelos desengordurados, obtidos a partir das tortas de 
extração (ultrassom na melhor condição de extração e prensa), sendo os coagulantes denominados respectivamente de coagulante integral, coagulante ultrassom e coagulante prensa. Inicialmente realizou-se uma lavagem da torta proveniente do método de ultrassom com água a $60^{\circ} \mathrm{C}$ e secagem também a $60^{\circ} \mathrm{C}$ para retirada do solvente residual.

Para o preparo dos coagulantes utilizouse $1 \mathrm{~g}$ de semente para $0,1 \mathrm{~L}$ de solução de $\mathrm{NaCl} 1 \mathrm{M}$. A semente integral, farelo desengordurado do ultrassom e da prensa foram triturados em liquidificador por 3 minutos, em seguida foram agitados em agitador magnético durante 30 minutos e, finalmente, filtrados com papel de filtro qualitativo (MADRONA et al., 2010).

\subsection{Análise de Proteína}

O método de Lowry et al. (1951) foi utilizado para quantificação das proteínas totais presentes nos coagulantes integral, coagulante ultrassom e coagulante prensa.

\subsection{Ensaios de Coagulação/Floculação}

2.4.1 Caracterização da Água Bruta

Foram avaliados os parâmetros de cor, compostos com absorção em $\mathrm{UV}_{254 \mathrm{~nm}}$ (espectrofotômetro DR 5000 Hach), turbidez (turbidímetro 2100P Hach) e pH (pHmetro Thermo-Scientific VSTAR92 Orion Versastar) segundo metodologia descrita no Standard Methods (APHA, 2005).

\subsubsection{Ensaios de Coagulação/Floculação}

Os coagulantes foram avaliados no processo de coagulação/floculação em ensaios em Jar test simples, Milan- Modelo JT 101/6 de seis provas. As condições operacionais do Jar test foram de tempo de mistura rápida de 3 minutos com gradiente de mistura rápida de $100 \mathrm{rpm}$ e tempo de mistura lenta de 15 minutos com gradiente de mistura de $15 \mathrm{rpm}$ (MADRONA et al., 2012), seguido de 30 minutos de decantação ao final deste processo.
As dosagens dos coagulantes avaliados foram calculadas com base na concentração de proteína presente em cada solução, utilizando-se uma concentração de 13,78 $\mathrm{mg} / \mathrm{L}$ de proteína, escolhida com base em estudo desenvolvido por Baptista et al. (2015). Para verificar o processo de remoção dos parâmetros se comparou os valores de cor, turbidez e $\mathrm{UV}_{254 \mathrm{~nm}}$ da água bruta com o obtido na água após seu tratamento.

\subsubsection{Carbono orgânico total (COT)}

Para a determinação do COT, primeiramente as amostras passaram por um processo de filtração em membrana de éster de celulose da Millipore, com diâmetro de poro médio $0,45 \mu \mathrm{m}$ com o auxílio de uma bomba a vácuo. Posteriormente, a concentração de COT (mg/L) foi determinada no equipamento TOC Analyser, da marca Shimadzu, modelo $5000^{\mathrm{a}}$, segundo procedimento descrito no Standard Methods (APHA, 2005). A análise de carbono orgânico total foi realizada nos coagulantes e nas águas após o tratamento com os mesmos.

\section{RESULTADOS E DISCUSSÃO}

\subsection{Extração do óleo}

A Tabela 1 apresenta os dados para a extração do óleo de Moringa utilizando o método do ultrassom. Observa-se pela Tabela 1 que o aumento no volume de solvente implica em um maior teor de óleo obtido, no entanto para as razões de massa: volume superior a 1:12 e tempo de extração acima de 60 minutos já não há diferença significativa, ao nível de significância de 5\%, no teor de óleo recuperado. Por outro lado, o tempo de extração apresentou uma menor influência na extração do óleo das sementes. 
Tabela 1: Teor de óleo $(\%)^{*}$ da semente de Moringa por extração utilizando ultrassom.

\begin{tabular}{ccc}
\hline $\begin{array}{c}\text { Razão Massa: } \\
\text { Volume }\end{array}$ & Tempo (min) & Teor de Óleo \\
\hline $1: 8$ & 30 & $22,02^{\mathrm{a}} \pm 0,89$ \\
$1: 8$ & 60 & $26,49^{\mathrm{b}} \pm 0,21$ \\
$1: 8$ & 90 & $27,25^{\mathrm{b}} \pm 0,26$ \\
$1: 12$ & 30 & $26,63^{\mathrm{b}} \pm 0,20$ \\
$1: 12$ & 60 & $32,66^{\mathrm{c}} \pm 0,15$ \\
$1: 12$ & 90 & $32,85^{\mathrm{c}} \pm 0,10$ \\
$1: 16$ & 30 & $32,56^{\mathrm{c}} \pm 0,10$ \\
$1: 16$ & 60 & $32,85^{\mathrm{c}} \pm 0,61$ \\
$1: 16$ & 90 & $32,49^{\mathrm{c}} \pm 0,47$ \\
\hline
\end{tabular}

*Teor de óleo $(\%)=$ (massa de óleo/massa de semente)*100. Letra diferente na mesma coluna indica diferença significativa $(\mathrm{p} \leq 0,05) .(\mathrm{M} \pm \mathrm{SD})$ média \pm desvio padrão.

Estudos relatam que para as extrações com o solvente hexano utilizando o aparelho soxhlet os teores de óleo obtido estão entre 40 e $30,8 \%$ (NZIKOU et al., 2002; ABDULKARIM et al., 2005). No entanto, no processo convencional de extração com o aparelho Soxhlet são utilizados maiores proporções de solvente e um tempo de extração total de oito horas. Resultados semelhantes foram obtidos para a semente de uva e linhaça (DA PORTO et al., 2013; ZHANG et al., 2008).

Considerando a minimização com gastos relacionados principalmente a energia e a não diferença apresentada pelas amostras, a condição mais adequada para a extração do óleo se apresenta como a razão massa de semente/volume de solvente de 1:16 e tempo de extração de 30 minutos com um o percentual de 32,56\%. Assim, optou-se por desengordurar o óleo nestas condições, assim como avaliar as suas características.

A Tabela 2 apresenta as características químicas dos óleos de Moringa obtidos pelos processos de ultrassom na melhor condição obtida, tempo de extração (30 minutos) e massa de semente: volume de solvente (1:16) e processo mecânico (prensa), onde se observa que houve diferença entre as amostras quanto ao teor de óleo e índice de acidez.

O teor de óleo obtido para a extração com a metodologia do ultrassom apresentou valores superiores $(32,56 \%)$ em relação ao obtido por prensa hidráulica $(26,78 \%)$, além disso, a extração empregando ultrassom mostrou uma grande economia no tempo de operação. Silva et al. (2013) obteve para a extração com hexano em equipamento de soxhlet, em um tempo de extração de 8 horas e proporção massa de semente: volume de solvente 1:30, um teor de óleo de $37 \%$. Desta maneira, pode-se dizer que o processo por ultrassom se mostra mais vantajoso que os processos utilizando o soxhlet e a prensa, para a obtenção do óleo de Moringa. Este método se destaca pela reprodutibilidade, rapidez do processo e redução da quantidade de solvente, sendo, portanto um processo ambientalmente mais favorável, em comparação com outros métodos que utilizam solventes orgânicos.

Tabela 2: Características químicas do óleo de semente de Moringa obtido por extração com ultrassom e prensa.

\begin{tabular}{c|c|c}
\hline Parâmetro & $\begin{array}{c}\text { Óleo de } \\
\text { Moringa/ } \\
\text { ultrassom }\end{array}$ & $\begin{array}{c}\text { Óleo de } \\
\text { Moringa/ } \\
\text { prensa }\end{array}$ \\
\hline $\begin{array}{c}\text { Teor de óleo } \\
(\%)\end{array}$ & $32,56^{\mathrm{a}} \pm 0,10$ & $26,78^{\mathrm{b}}$ \\
$\begin{array}{c}\text { Tempo de } \\
\text { Extração } \\
(\text { min) } \\
\text { Indice de }\end{array}$ & 30 & 120 \\
$\begin{array}{c}\text { acidez (\% de } \\
\text { ácido oléico) } \\
\text { Índice de }\end{array}$ & $0,54^{\mathrm{a}} \pm 0,30$ & $0,44^{\mathrm{b}} \pm 0,01$ \\
$\begin{array}{c}\text { saponificação } \\
(\text { mg KOH/g) }\end{array}$ & $217,22^{\mathrm{b}} \pm 1,73$ & $164,87^{\mathrm{b}} \pm 1,25$ \\
\hline
\end{tabular}

Letras diferentes na mesma linha indicam diferença significativa $(\mathrm{p}<0,05)$. 
Os resultados mostram valores mais elevados de índice de acidez e saponificação para o óleo obtido por ultrassom. No entanto, os valores de índice de acidez $(0,54 \%$ ácido oleico) ainda está abaixo dos valores encontrados na literatura $(0,82$ a 2,48 \% ácido oleico) (TSAKINS et al., 1998; ABDULKARIN et al., 2005) para a extração utilizando o solvente hexano no aparelho soxhlet. Os valores do índice de saponificação para o processo de ultrassom estão acima do esperado, sendo necessário o estudo de processos brandos de refino, o inverso ocorreu com o processo mecânico.

\subsection{Ensaios de coagulação/floculação}

A água bruta utilizada nos ensaios de coagulação/floculação apresentou parâmetros médios de $\mathrm{pH} 7,98$, cor de 463,5 $\mathrm{mgPtCo} / \mathrm{L}$, turbidez de 98,80 NTU e $\mathrm{UV}_{254 \mathrm{~nm}}$ de 0,359 $\mathrm{cm}^{-1}$. Os resultados de remoção de cor, turbidez e $\mathrm{UV}_{254 \mathrm{~nm}}$ obtidos com o uso do coagulante da semente integral, coagulante ultrassom e coagulante prensa são apresentados na Figura 1.

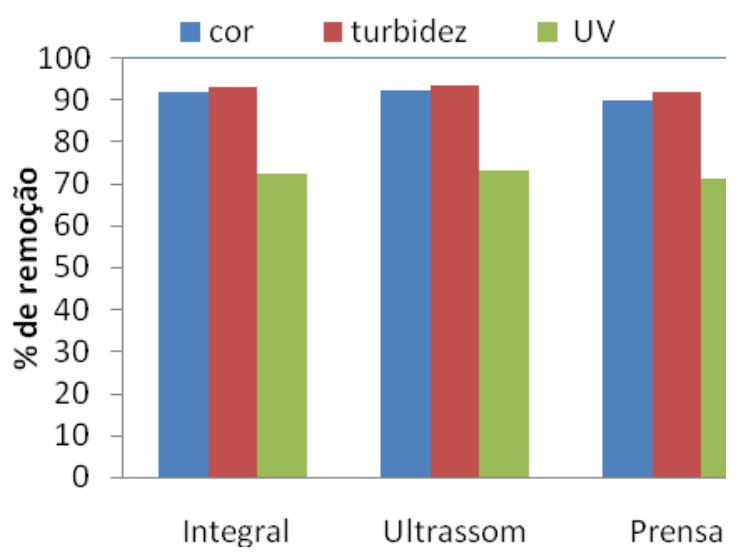

Figura 1 - Porcentagem de remoção de cor, turbidez e $\mathrm{UV}_{254 \mathrm{~nm}}$ utilizando o coagulante integral, coagulante ultrassom e coagulante prensa.

Ao observar a Figura 1, pode-se verificar que os coagulantes apresentaram percentuais de remoção dos parâmetros similares entre si. Com o uso do Coagulante Integral, Ultrassom e Prensa foi possível obter, respectivamente, porcentagens de remoção de $92,02 \%, 92,45 \%$ e $90,19 \%$ para cor, e valores para turbidez de 93,15\%, $93,55 \%$ e $92,2 \%$. Resultado semelhante foi encontrado por Ndabigengesere e Narasiah (1998) que obtiveram uma remoção de $90 \%$ da turbidez de água bruta com 105 NTU, porém utilizando uma dosagem de coagulante de $50 \mathrm{mg} / \mathrm{L}$, valor bem superior ao utilizado no presente estudo, o qual foi de $13,78 \mathrm{mg} / \mathrm{L}$ de proteína.

Os compostos com absorção em $\mathrm{UV}_{254 \mathrm{~nm}}$ apresentaram percentuais de remoção inferior ao encontrado para cor e turbidez, com remoções de $72,55 \%, 73,4 \%$ e $71,45 \%$, respectivamente para o Coagulante Integral, Ultrassom e Prensa. A absorbância em luz $\mathrm{UV}_{254 \mathrm{~nm}}$ auxilia na estimativa do conteúdo de matéria orgânica contida na amostra (PENITSKY, 2003), e pelo fato do coagulante de Moringa ser orgânico torna-se mais difícil obter altos valores de remoção deste parâmetro. Os percentuais de remoção de $\mathrm{UV}_{254 \mathrm{~nm}}$ encontrados foram superiores ao obtidos por Madrona et al. (2012) que conseguiu um percentual de $47 \%$ na remoção deste parâmetro em uma dosagem de coagulante de $25 \mathrm{mg} / \mathrm{L}$ no tratamento de água de alta turbidez. Desta forma, estes resultados indicam que o modo de extração do óleo não afeta a capacidade de coagulação/floculação do coagulante obtido da Moringa.

\subsection{Carbono orgânico total}

A análise do teor de carbono orgânico total (COT) foi realizada no Coagulante Integral, Coagulante Ultrassom, Coagulante Prensa, nas águas tratadas com estes coagulantes e seus resultados são apresentados na Tabela 3 . 
Tabela 3: Carbono orgânico total (COT) encontrado nos coagulantes, nas águas tratadas e na água bruta.

\begin{tabular}{c|c}
\hline Amostra & COT $(\mathrm{mg} / \mathrm{L})$ \\
\hline Coagulante Integral & 1857,00 \\
Coagulante Ultrassom & 1795,00 \\
Coagulante Prensa & 2269,00 \\
Água tratada com & 7,38 \\
coagulante integral & Água tratada com \\
coagulante ultrassom & 4,98 \\
$\begin{array}{c}\text { Água tratada com } \\
\text { coagulante prensa } \\
\text { Água bruta }\end{array}$ & 17,74 \\
\hline
\end{tabular}

Os resultados apresentados na Tabela 3 demonstram que, dentre os coagulantes avaliados, o Coagulante Ultrassom apresenta o menor teor de COT, inclusive menor do que o Coagulante integral. Este resultado se reflete também na água tratada, sendo que a água tratada com o Coagulante Ultrassom apresentou o menor teor de carbono orgânico total, o qual foi de $4,98 \mathrm{mg} / \mathrm{L}$. o coagulante obtido com a prensa foi o que apresentou os piores resultados, com uma matéria orgânica residual, na água tratada, de $17,74 \mathrm{mg} / \mathrm{L}$. Neste caso, o processamento da semente por prensa pode ter deixado o óleo contido nas sementes mais livre devido ao atrito mecânico inerente ao próprio processo, além de que no processo da prensa houve uma menor extração de óleo quando comparado à extração por ultrassom conforme verificado anteriormente.

Desta forma, pode-se dizer que a utilização do Coagulante Ultrassom é vantajosa, pois seu uso acarretou em diminuição do COT, que partiu de uma concentração de $5,78 \mathrm{mg} / \mathrm{L}$ na água bruta para $4,98 \mathrm{mg} / \mathrm{L}$ após o tratamento. Sabe-se que uma menor quantidade de matéria orgânica na água tratada diminui problemas com sabor, odor e o desenvolvimento de microrganismos durante o armazenamento, além de reduzir o risco de formação de trihalometanos durante o processo de desinfecção por cloro, substância esta prejudicial à saúde por ser cancerígena (MUYIBI, 1995).

\section{CONCLUSÃO}

A semente de Moringa oleifera apresenta potencial para sua utilização como fonte de óleo vegetal de boa qualidade. Os resultados mostram um maior teor de óleo para a extração por ultrassom $(32,56 \%)$ em comparação com o processo mecânico $(26,78 \%)$.

A avaliação dos processos de coagulação/floculação mostra que não há diferença em relação a origem do coagulante, se integral, desengordurado por ultrassom ou por prensa, quanto à remoção de cor, turbidez e $U_{254 n m}$. Porém, o Coagulante Ultrassom se mostrou mais eficiente contribuindo para a diminuição da matéria orgânica ao final do processo de tratamento da água.

\section{BIBLIOGRAFIA}

ABDULKARIM, S.M., LONG, K., LAI, O.M., MUHAMMAD, S.K.S. AND GHAZALI, H.M. Some physico-chemical properties of Moringa oleifera seed oil extracted using solvent and aqueous enzymatic methods. Food Chem. 93:253263, 2005.

ANWAR, F., HUSSAIM, A.I., IQBAL, S. AND BHANGER, M. Enhancement of the oxidative stability of some vegetable oils by blending with Moringa oleifera oil. Food Chem.103:1181-1191, 2007.

APHA - American Public Health Association. Standard methods for the examination for 
water and wastewater. 20th ed. Washington, D.C, 2005.

AOAC-Association Of Official Analytical Chemists . Official Methods of analysis of the Association of Official Analytical Chemists. $16^{\text {th }}$ Ed. Washington, D.C, 1998.

AYERZA, R. Seed yield components, oil content, and fatty acid composition of two cultivars of moringa (Moringa oleifera Lam.) growing in the Arid Chaco of Argentina. Ind. Crop. Prod. 33:389-394, 2011.

BAPTISTA, A. T. A., COLDEBELLA, P. F., CARDINES, P. H. F., GOMES, R. G., VIEIRA, M. F., BERGAMASCO, R., VIEIRA, A. M. S. 2015. Coagulationflocculation process with ultrafiltered saline extract of Moringa oleifera for the treatment of surface water. Chem. Eng. J. 276:166-173, 2015.

DA PORTO, C.; PORRETTO, E.; DECORTI, D.; Comparison of ultrasound-assisted extraction with conventional extraction methods of oil and polyphenols from grape (Vitis vinifera L.) seeds, Ultrasonics Sonochemistry. 20:1076-1080, 2013.

KABORE, A., SAVADOGO, B., ROSILLON, F., TRAORE, A., DIANOU, D., Effectiveness of Moringa oleifera Defatted Cake versus Seed in the Treatment of Unsafe Drinking Water: Case Study of Surface and Well Waters in Burkina Faso. J. Water Resour. Prot. 5:1076-1086 2013.

LOWRY, O.H., ROSEBROUGH, N.J., FARR, A.L.; RANDALL, R. J. J. Biol. Chem. 193:265, 1951.

MADRONA, G. S., SERPELLONI, G. B., VIEIRA, A. M. S.; NISHI, L., CARDOSO, K. C., BERGAMASCO, R. Study of the effect of saline solution on the extraction of the Moringa oleifera seed's active component for water treatment. Water, Air, \& Soil Poll. 211:409-415, 2010.

MADRONA, G.S., BRANCO, I.G., SEOLIN, V.J., ALVES FILHO, B. DE A., FAGUNDES-KLEN, M.R., BERGAMASCO, R. Evaluation of extracts of Moringa oleifera Lam seeds obtained with $\mathrm{NaCl}$ and their effects on water treatment. Acta Sci. Technol. 34:289-293, 2012..

MENEZES, M. L., DANILUSSI, M. S., BARROS, S. T. D.; PEREIRA, N. C. Extração do óleo de semente de uva por ultrassom (extração utilizando o ultrassom). XXXVI Congresso de Sistemas Particulados, 2013.

MUYIBI, S.A., EVISON L.M. Optimizing Physical Parameters affecting coagulation of turbid water with Moringa oleifera seeds. Water Res. 29:2689-2695, 1995.

NDABIGENGESERE, A., NARASIAH, K.S. Quality of Water Treated by Coagulation Using Moringa oleifera Seeds. Water Res. 32: $781-791,1998$.

NISHI, L., MADRONA, G. S., GUILHERME, A. L. F., VIEIRA, A. M. S., ARAÚJO, A. A., UGRI, M. C. B. A., BERGAMASCO, R.. Cyanobacteria removal by coagulation/floculation with seeds of the natural coagulant Moringa oleifera Lam. Chem. Eng.Trans. 24:11291134, 2011

PENITSKY, D.J. Coagulation 101" in Proceedings Technology Transfer Conference, Universidade de Calgary, Alberta, Canadá, 2003.

TSAKNIS, J., LALAS, S., GERGIS, V., SPILIOTIS $\mathrm{V}$. A total characterisation of 
Moringa oleifera Malawi seed oil. Riv. Ital. Sost. Gras. 75:21-27, 1998.

SILVA, M. O., FAVERO, C. F., LAZARI, J. P., VIEIRA, M. F., BONAFE, E., VISENTAINER, J. V., PEREIRA, N. C., BERGAMASCO, R., VIEIRA, A. M. S. Some Chemical Characterization Oil of Seed Moringa Oleifera Obtained by Solvent and Mechaniical Extraction, 11th Euro Fed Lipid Congress, Antalaya, 2013.

VIEIRA, A. M. S., VIEIRA, M. F., SILVA, G. F., ARAÚJO, A. A., FAGUNDES-KLEN, M. R., VEIT, M. T.; BERGAMASCO, R. Use of Moringa oleifera Seed as a Natural Adsorbent for Wastewater Treatment. Water, Air, \& Soil Poll. 206: 273-281, 2010

ZHANG, Z.; WANG L., LI, D., JIAO, S.; CHEN X. D.; MAO, Z. Ultrasound-assisted extraction of oil from flaxseed, Separation and Purification Technology vol. 62 , 192198, 2008.

\section{AGRADECIMENTOS}

Os autores agradecem ao apoio financeiro recebido pelo Conselho Nacional de Desenvolvimento Científico e Tecnológico (CNPQ), Coordenação de Aperfeiçoamento de Pessoal de Nível Superior (CAPES) e a Fundação Araucária. 